\title{
Scalable boson encoding for VQE
}

\section{Andy C. Y. Li, Alexandru Macridin, Panagiotis Spentzouris \\ Fermi National Accelerator Laboratory}

\section{Highlights}

- Number-basis binary encoding with scalable measurement scheme for boson operators

- Demonstration using a Rabi model VQE application

\section{Number basis binary encoding}

Goal: encode the states in a truncated boson Hilbert space by a finite number of qubits using the number basis

$$
\begin{aligned}
& \text { Number basis encoding } \\
& |\phi\rangle=\sum_{n=0}^{N} \phi_{n}|n\rangle \\
& |n=N\rangle=|1 \ldots 11\rangle_{q} \\
& |n=3\rangle=|0 \ldots 11\rangle_{q} \\
& |n=2\rangle=|0 \ldots 10\rangle_{q} \\
& |n=1\rangle=|0 \ldots 01\rangle_{q} \\
& |n=0\rangle=\underbrace{|0 \ldots 00\rangle_{q}} \\
& q_{c} \text { qubits } \\
& \mathrm{n}=\sum_{j=0}^{q_{c}-1} 2^{q_{c}-j-1} \mathrm{n}_{j}^{q} \\
& \mathrm{a}^{\dagger}=\sum_{j=0}^{q_{c}-1} 2^{\frac{q_{c}-1}{2}} \sqrt{\sum_{k=0}^{j-1} \frac{\mathrm{n}_{k}^{q}}{2^{k}}+\frac{1}{2^{j}}} \sigma_{j}^{+} \prod_{k=j+1}^{q_{c}-1} \sigma_{k}^{-} \\
& \mathrm{a}=\sum_{j=0}^{q_{c}-1} 2^{\frac{q_{c}-1}{2}} \sqrt{\sum_{k=0}^{j-1} \frac{\mathrm{n}_{k}^{q}}{2^{k}}+\frac{1}{2^{j}}} \sigma_{j}^{-} \prod_{k=j+1}^{q_{c}-1} \sigma_{k}^{+}
\end{aligned}
$$

Measure $\langle f(\vec{q}, \vec{p})\rangle$ with $N_{I}$-boson-mode interaction where $f$ is a polynomial function of degree $n_{f}$

Qubit count per mode

$$
q_{c}=\log _{2}(N+1)
$$

Circuit sampling count $\leq O\left(q_{c}^{N_{I} \operatorname{ceil}\left(1+\log _{2} n_{f} / N_{I}\right)}\right)$

Gate count

$$
O\left(N_{I} \times q_{c}\right)
$$

\section{- Logarithmic memory efficiency}

- Shallow circuit depth for expectation value measurement

- Practical circuit sampling overhead

- Suitable for VQE applications of multi-mode systems involving a large number of bosons

$f(\mathrm{n})\rangle$

$Q_{0} \longrightarrow$ Z

$|\phi\rangle \quad Q_{1}$

$$
Q_{q_{c}-1}
$$

Measurement circuit: $\mathrm{CX}_{j k} \sigma_{j}^{+} \sigma_{k}^{-} \mathrm{CX}_{j k}=\sigma_{j}^{+} n_{k}^{q}$

$U \sigma_{j}^{+} \prod_{k=j+1}^{q_{c}-1} \sigma_{k}^{-} U$ $=\sigma_{j}^{+} \prod_{k=j+1}^{q_{c}-1} n_{k}^{q}$ $\langle f(\mathrm{q}, \mathrm{p})\rangle$
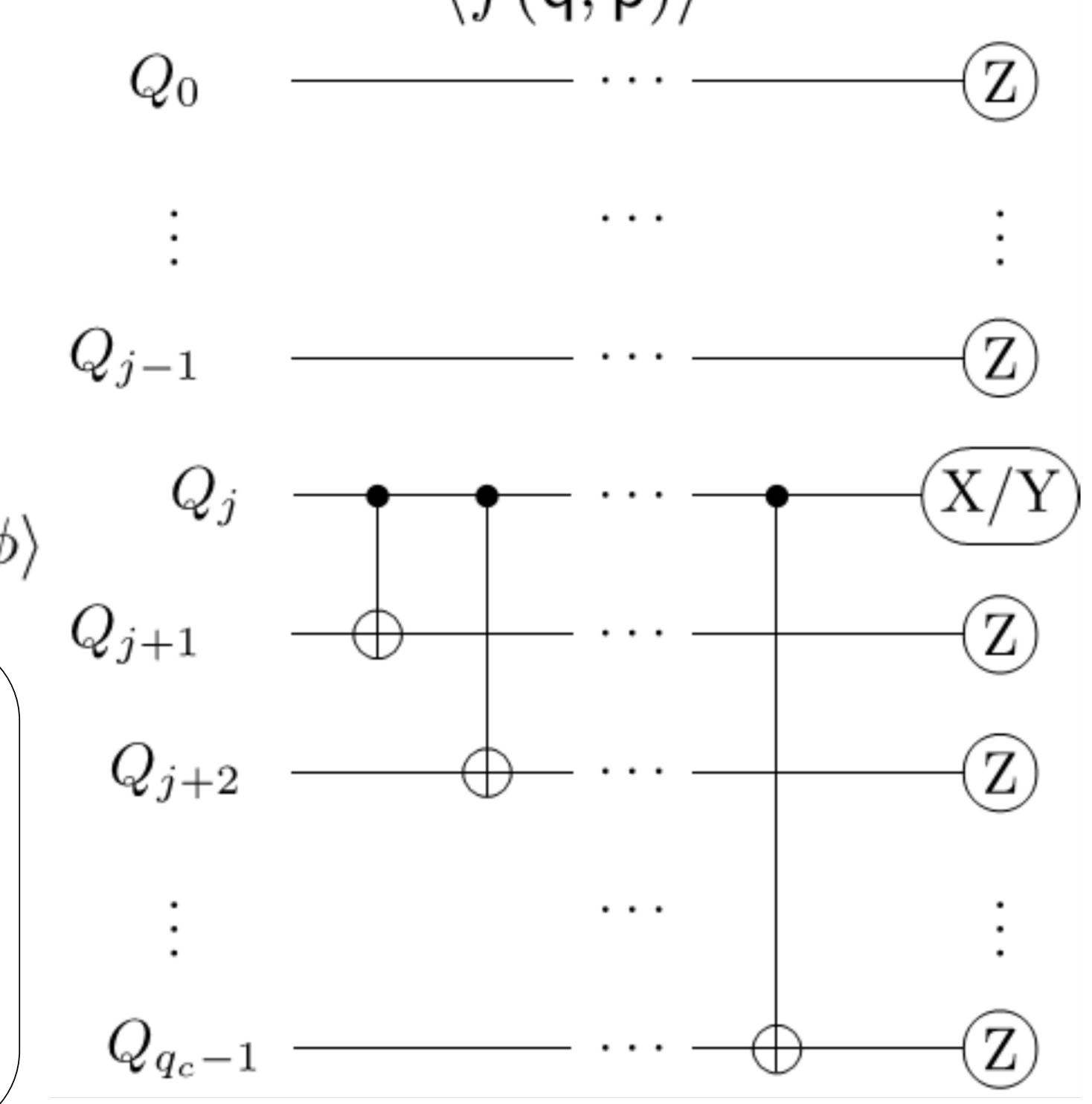

\section{VQE of Rabi model with Rigetti's Aspen}

Rabi model - simple light-matter interaction

$$
H=\Omega a^{\dagger} a+\frac{\Omega}{2} \sigma_{z}+\frac{g}{2}\left(a^{\dagger}+a\right) \sigma_{x}
$$

The photon mode (truncated to up to three photons) is encoded using two qubits.

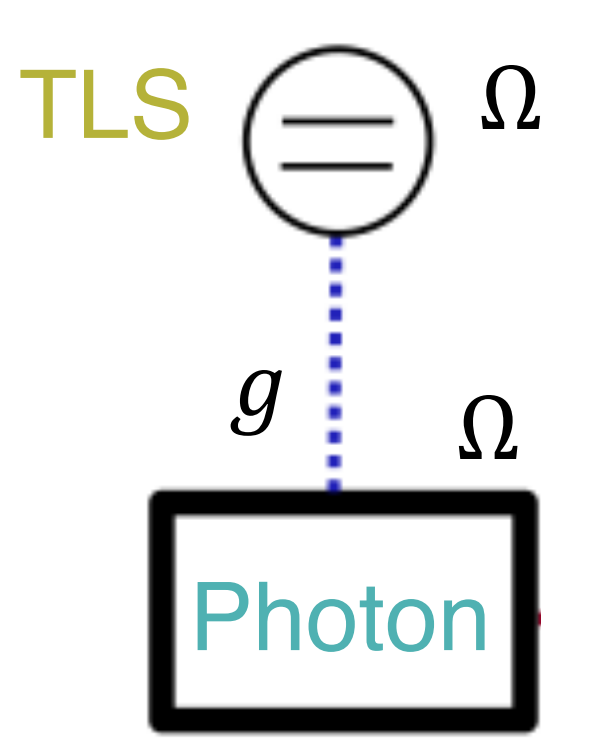

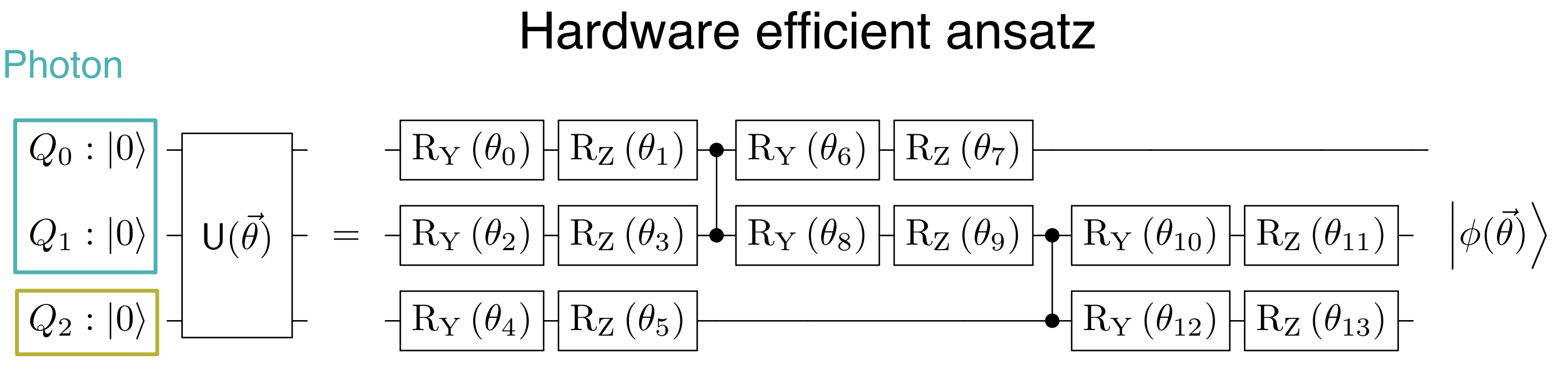

TLS

Optimization for low-energy spectrum

Cost function: $C_{j}=\langle\psi(\vec{\theta})|H| \psi(\vec{\theta})\rangle+p \sum_{k=0}^{j-1}\left|\left\langle\psi_{k} \mid \psi(\vec{\theta})\right\rangle\right|^{2}$

Eigenstates: $\left|\psi_{j}\right\rangle=\underset{|\vec{\theta}\rangle}{\operatorname{argmin}} C_{j} \quad$ Ref: O. Higgott, D. Wang, and

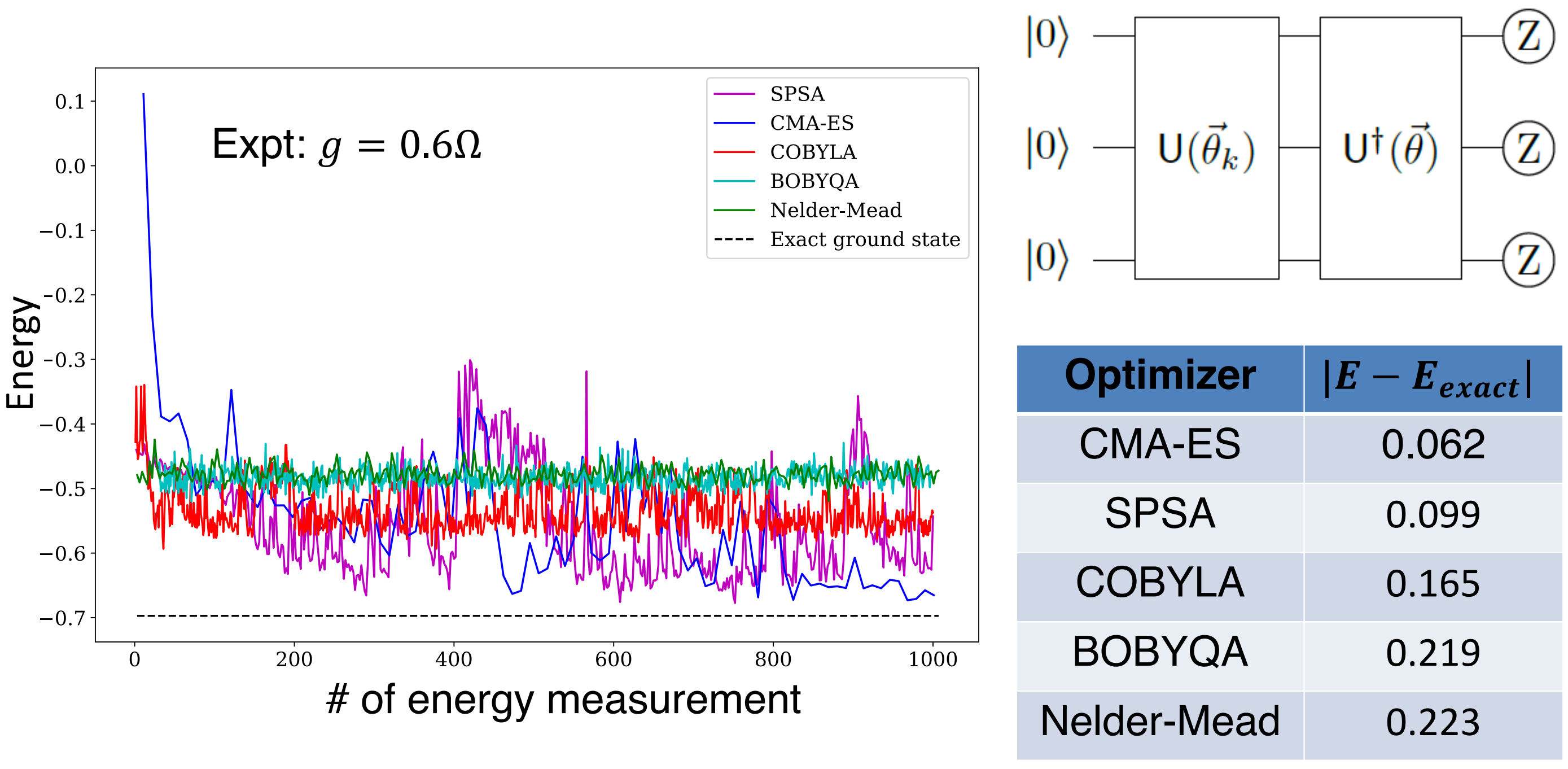

- Low-energy spectrum: ground and first-excited states

- Narrowing of energy gap with increasing coupling $g$

- Discrepancy between experimental result and exact solution: hardware noise and sampling errors

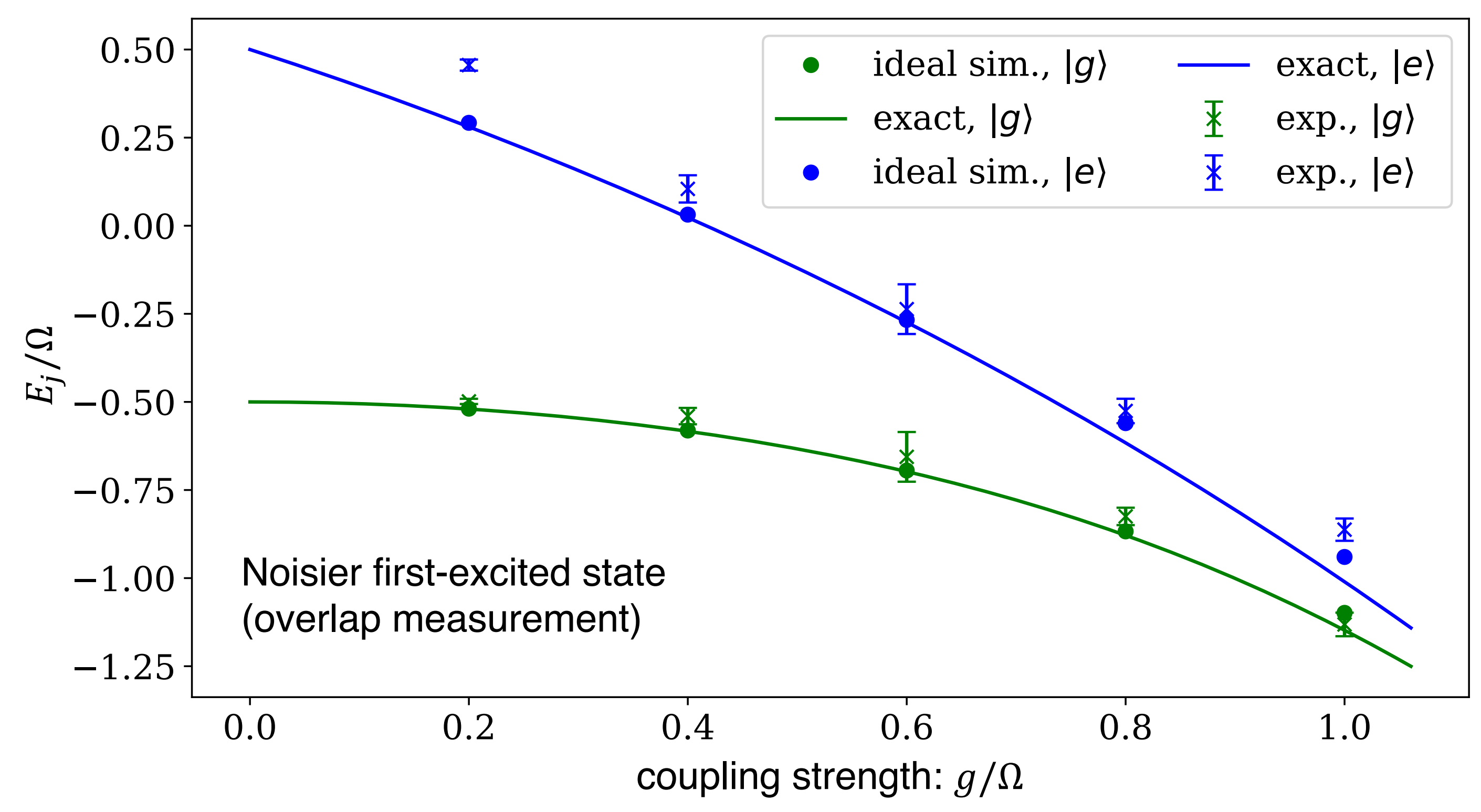

This Poster has been authored by Fermi Research Alliance, LLC under Contract No. DE-AC02-07CH11359 with the U.S. Department of Energy, Office of Science, Office of High Energy Physics. 